Classification

Physics Abstracts

07.05.P $-06.20-07.60$

\title{
An Image Analysis Based Full-Embedded System for Optical Metrology
}

\author{
Richard Grisel $\left({ }^{1}\right)$, Nicolas Giraud $\left({ }^{1}\right)$, Ahmed Haddadi $\left({ }^{2}\right)$ and Michel Jourlin $\left({ }^{1}\right)$ \\ $\left({ }^{1}\right)$ CPE-LISA (CNRS EP 0092), 43 boulevard du 11 Novembre 1918, \\ 69616 Villeurbanne Cedex, France \\ $\left({ }^{2}\right)$ ESSILOR International, Instruments Dpt, 1 rue Thomas Edison, 94028 Créteil Cedex, France
}

\begin{abstract}
Résumé. - Nous présentons un système d'analyse d'images dédié à la mesure automatique de paramètres en métrologie optique. Larticle décrit les aspects théoriques et pratiques de la mise en oeuvre, en insistant sur les techniques utilisées pour obtenir une précision de $0,5 \mathrm{~mm}$ sur les mesures effectuées.
\end{abstract}

\begin{abstract}
A full-embedded system for automatic measurement in optical metrology is presented. This paper deals with the theoretical and practical aspects of the construction of the system, emphasizing methods used to reach a $0.5 \mathrm{~mm}$ precision for the measurements obtained by the image analysis processing.
\end{abstract}

\section{Introduction}

The process deals with optical metrology, more precisely the measure of different parameters required for making glasses frame. The opticians need to cut the lenses according to the frame pattern and to the different parameters related to the patient (see Fig. 1). Actually, the objective is to match the eye centre with the lens optical centre. This operation is called lens centring. Many optical metrology processes exist. Classically, opticians measure on the patient the eye centre position within the pattern with a reglet. This method is not really accurate because the patient head position is not natural. To palliate this drawback, new devices integrate a video camera linked to a PC digitalizing patient's face image. Whereas the use of a PC mouse is easy for parameters determination, automatic extraction of frame glasses on the patient's face is a far more complex task requiring visual perception ability and intelligent interpretation. Recent work has been done on this subject. Bouyanzer [1] launched a study based on gradient [2-4] and adaptive thresholding algorithms [5]. We tried to implement new algorithms using compass gradient edge operators [6] and mathematical morphology [7-9]. 


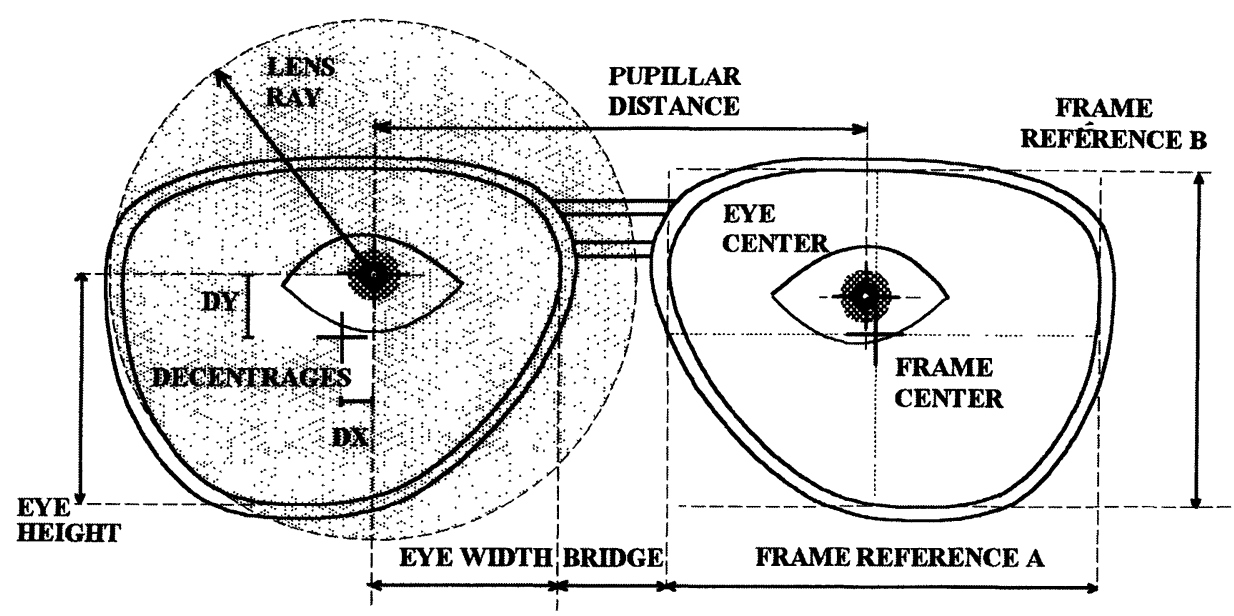

Fig. 1. - Parameters needed for lens centring. The $(\mathrm{d} y, \mathrm{~d} x)$ parameters obtained by the computation of eye-centre, height and width allow to make glasses frame.

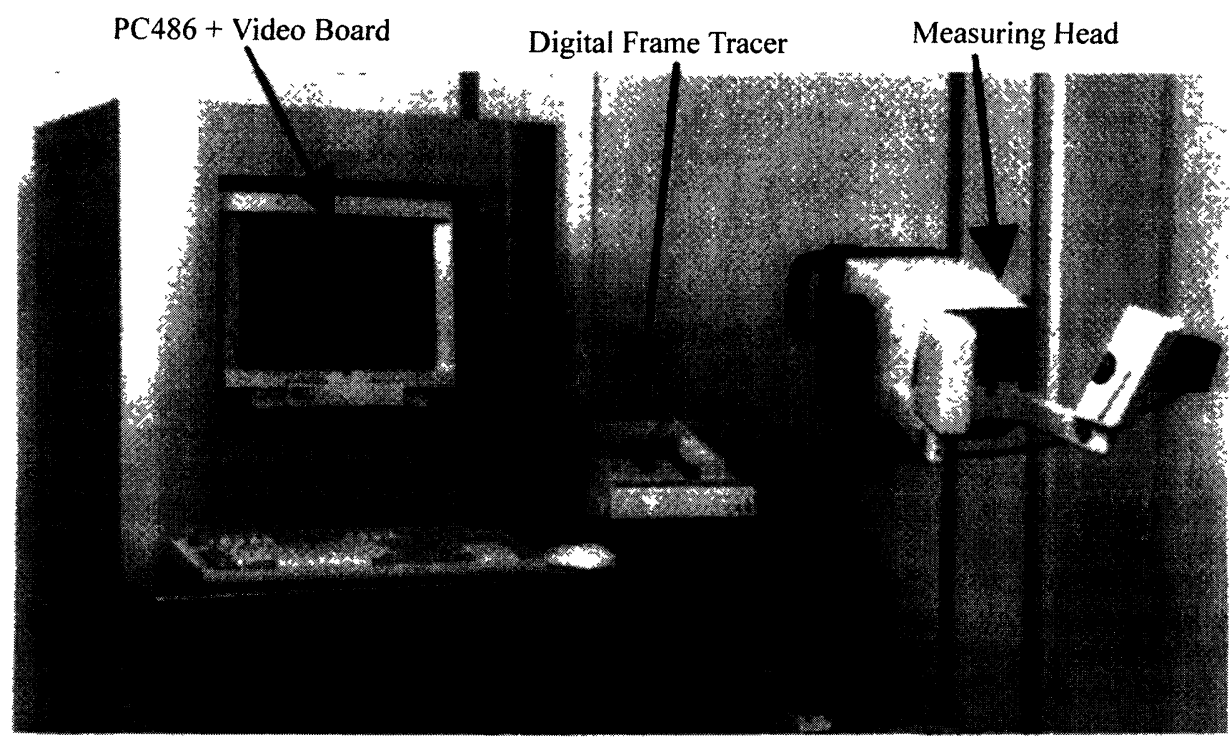

Fig. 2. - Digital frame tracer, measuring head and PC-486 with video acquisition board. This is the overview of the full-embedded system.

\section{System Description}

The system, presented in Figure 2, is a measurement device in the field of optical metrology. It should ensure reproducible results respecting the physiological conditions. To respect natural head position, a mechanical balanced arm allows to set the measuring head in front of the patient without touching him. 


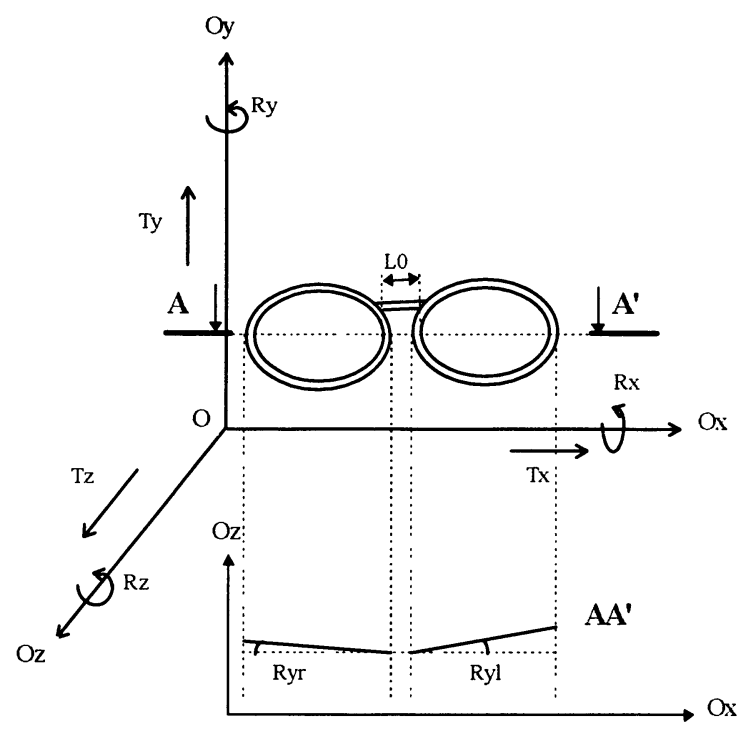

Fig. 3. - Localization of video cameras/patient's frame translations $\left(\mathrm{T}_{x}, \mathrm{~T}_{y}, \mathrm{~T}_{z}\right)$ and rotations $\left(\mathrm{R}_{x}, \mathrm{R}_{y 1}, R_{y \mathrm{r}}, R_{z}\right)$. Two cameras are centred on each eye ( $80 \mathrm{~mm}$ optical field), the third is lateral to obtain lens/eye distance.

Optical System. - It has two goals. The first one is to set the measuring head to an accurate distance and orientation of the patient (see Fig. 3). $\mathrm{T}_{z}$ and $\mathrm{R}_{x}$ are controlled by lateral mirrors orientation $45^{\circ}$, distance $300 \mathrm{~mm}-\mathrm{T}_{y}$ and $\mathrm{T}_{x}$ are controlled by 2 small bulbs lighting the image centre. The second goal of the optical system is to fix patient's look. Diodes are placed inside the measuring head for this purpose.

Video SySTEM. - It is organized in 3 color video sources. Video camera resolution is $768 \times 493$ captors (CCD technology). The pixel size is $0.15 \mathrm{~mm}$. The choice of 2 video cameras on the front, centred on each eye, allows to decrease optical distortion by reducing each optical field to $80 \mathrm{~mm}$. The third camera is set on the lateral field to control $\mathrm{R}_{y}$ and $\mathrm{T}_{z}$ and to obtain lens/eye distance. In this way, stereological data are obtained. This process is covered by the Essilor patent $\mathrm{N}^{\circ} 9405400$ : "Procédé de métrologie optique".

Video system qualification was necessary for our application. A collaboration with the National Test Laboratory of France (LNE) was undergone to do 4 tests with video camera of COHU company: image uniformity, pulse response and horizontal edge spread function, blooming effect, geometrical distortions. All parameters needed for lenses centring will be given after a computation using the corrected distortion matrix.

\section{Image Processing}

3.1 Eye Centre Extraction. - When the patient watches a lighting, a reflection is created on the cornea. A $80 \times 60$ experimental size window is set around the expected eye position in order to accelerate the processing (see Fig. $4 \mathrm{a}$ ).

Eye centre determination has 3 steps: detection of a white spot (the reflection) on a dark area (the pupil), parasite reflections elimination, centroid computation. In our application, a Sobel 
a)

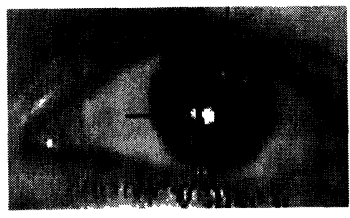

b)

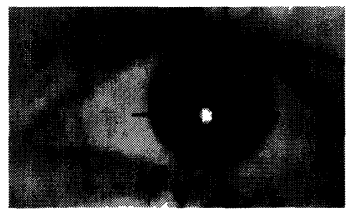

Fig. 4. - a) Detection of a white spot, $80 \times 60$ window around expected eye position. b) Parasite reflection elimination, Centroid computation for final $13 \times 13$ window.

gradient operator is applied. The neighbouring pixels (NP for short) are defined as the pixels which are covered by a $3 \times 3$ mask except the centre pixel $(i, j)$. Only the North directional mask of a 2-dimensional Sobel operator is applied to detect the vertical boundary between reflection and pupil. The edge value is the sum of the products of the weight of this mask and the intensity value of the corresponding NP. A $13 \times 13$ window centred on the detected frontier is set to enclose the reflections. Morphological filters [7, 9] were used since they are easy to implement (see Fig. 4b). The success rate for this process is $98 \%$.

3.2 Frame Extraction. - This process deals with the determination of the horizontal and vertical tangents within the frame. Knowing these parameters, the eye centre position can be computed in the frame reference. Two methods were investigated.

Tangent Extraction Based on Distance Histogram Threshold. - This method is based on the following steps.

- Gradient map computation. 4 processing windows (North, West, South, East) are set on each eye according to the eye centre position. The windows size $-[200,80]$ pixels for $\mathrm{N}$ and $\mathrm{S} /[80,200]$ for $\mathrm{E}$ and $\mathrm{W}-$ is determined to enclose any type of frames. 3 Prewitt operators $(3 \times 3)$ with 3 orientations $(\mathrm{N}, \mathrm{NW}, \mathrm{NE})$ are used to follow the frame curve, and the resulting image is obtained for each pixel by taking the maximum value.

- Gradient map threshold. One uses the cumulated histogram of the gradient map for which a variable threshold is defined as corresponding to the 2000 upper values of the cumulated histogram. The image becomes a binary image with pixel to 1 or 0 .

- Thickness and binarisation of contour lines. The algorithm used is based on comparison of the pixel with its two nearest neighbours using the following operation by example for $\mathrm{N}$ and S windows: $C(i, j)=0$ if $(C(i, j) \leq B(i, j-1)$ or $C(i, j) \leq B(i, j+1))$ else $B(i, j)$ with $C(i, j)=$ computed point for contour, $B(i, j)$ binary value from previous step.

- Tangent extraction. For each pixel, the distance from the point to the inside window border was computed. This value is reported into the distance histogram (see Fig. 5). A threshold, put at 5 pixels, is applied and the resulting tangent is the maximum value obtained. This algorithm allows also the computation of the glass diameter (see Fig. 6), and $\mathrm{R}_{z}$ angle (see Fig. 7). The success rates for this method are the followings:

Nasal and bottom windows: 90\%, temporal and upper window: $60 \%$ and 40\%; Glass diameter: $98 \%, \mathrm{R}_{z}$ detection: $98 \%$. The success rate is not sufficient mainly because faces (because of eyebrows, nose, lighting) and glasses frame (because of reflections and multiple colors) are not homogeneous areas. Thus an automatic extraction for optical metrology cannot be successful without a frame a priori knowledge. This is the purpose of the second method. 


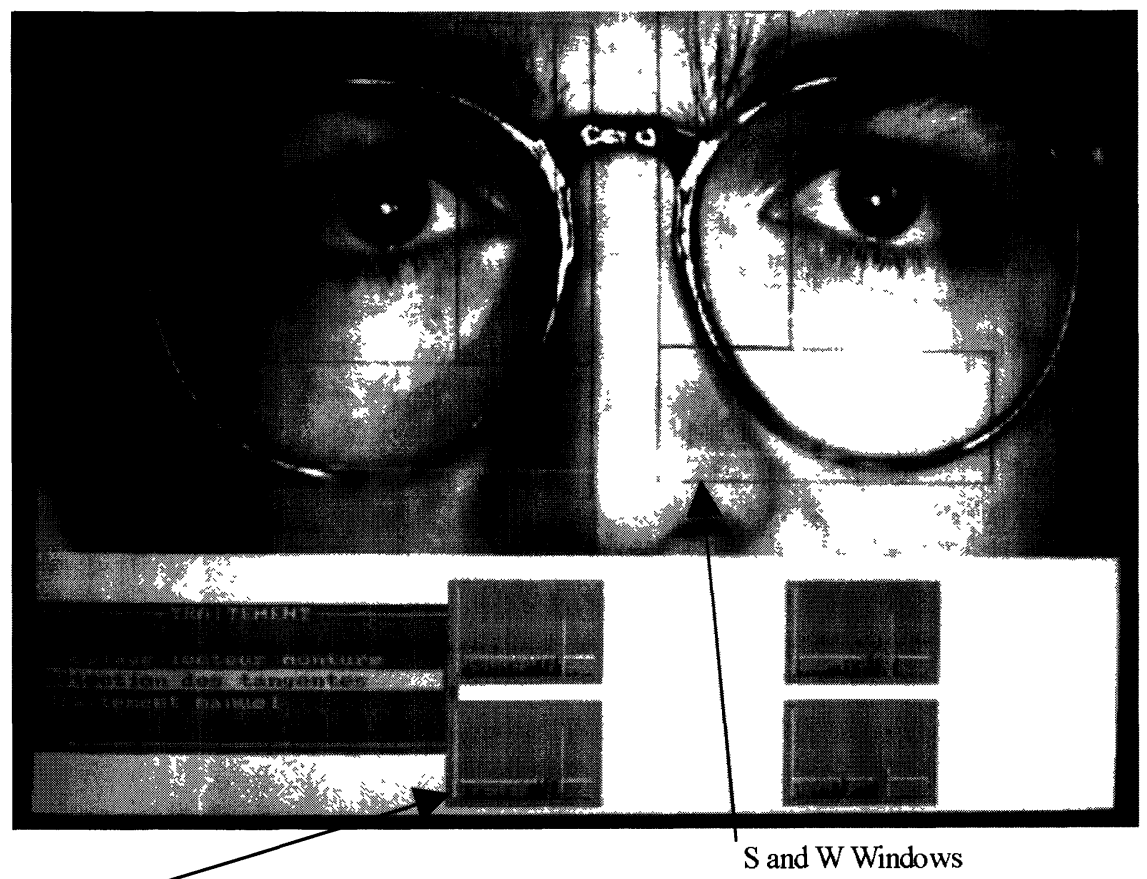

Distance Histogram Window : Number of pixels vs inside border distance The white horizontal line shows the threshold at 5 pixels the white vertical line shows the extracted tangent

Fig. 5. - Tangent detection.

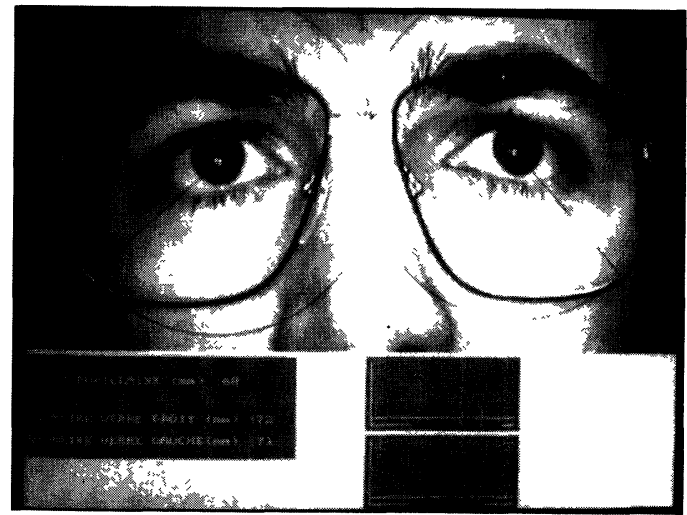

Fig. 6.

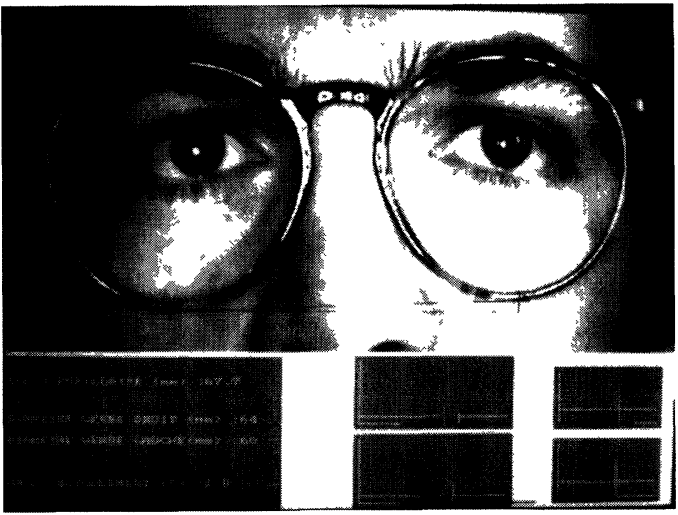

Fig. 7.

Fig. 6. - Glass diameter extraction by distance histogram. The circle shows the extracted diameter by overlay.

Fig. 7. $-\mathrm{R}_{z}$ angle extraction by distance histogram. The bottom line shows the extracted $\mathrm{R}_{z}$ angle. 
Model Matching Method. - We decided to use a file describing the frame slot and to match this information with the patient's image. This model is considered as the metrological reference for our processing. The matching operation is based not only on $\mathrm{T}_{x}$ and $\mathrm{T}_{y}$ translations but also on $\mathrm{T}_{z}$ translation and $\mathbf{R}_{x}, \mathbf{R}_{y}, \mathbf{R}_{z}$ rotations applied to the model. Also, the model is deformed to find frame features and optical parameters are obtained with the inverse geometrical deformation. Important work has been done on deformable templates [10,11]. Our study has some similarities with deformable templates described in [12] for faces features extraction. An important point of these works is the algorithm optimization by reducing model deformations and simplifying the representation of the image.

- Pre-processing. Our deformable model acts on only 1 representation of the image chosen to extract edges. The frame edges are extracted by a compass edge gradient operator [6]. 4 processing windows (North, West, South, East) are set on each eye according to the eye centre position. The windows size, [80,60] pixels for $\mathrm{N}$ and $\mathrm{S}$ and $[60,80]$ for $\mathrm{E}$ and $\mathrm{W}$, is determined to enclose any type of frames. At this point, both inner and external edges of the frame appear on the representation of the image. A specific threshold is applied to eliminate the external edge which could provide problems for our automatic matching operation. We observe the radial profile on the edge field from the eye centre to the outside. The Cartesian co-ordinates $p(i, j)$ in the edge field are translated into polar co-ordinates $p(\rho, \theta)$. The threshold applied for each angle corresponds to the first maximum detected. If the distance is greater than the threshold distance the pixel value on the edge field is set to 0 . This result is convolved with a smoothing operation on threshold distances for the neighbouring angles. All this pre-processing gives an easier matching process.

- Vectorization. The objective is to reduce the amount of information required to represent the content of the original frame file. The file consists of a set of $m$ points:

$$
F=\left\{\mathbf{P}_{j}=\left(x_{\jmath}, y_{j}\right) ; j=1, . ., m\right\} .
$$

The procedure identifies critical points of the model [13]. The first point $P_{1}$ is selected and belongs to the set CP of critical points:

$$
\mathrm{CP}=\left\{\mathrm{C}_{k}=\left(x_{k}, y_{k}\right) ; k=1, . ., q\right\} \quad \text { where } q<m \text { and } \mathrm{P}_{1}=\mathrm{C}_{1} .
$$

We denote by $\varepsilon$ the prescribed precision value. Let $\mathrm{P}_{i}=\mathrm{C}_{k-1}$ the ultimate labelled critical point. $\mathrm{P}_{j}$ belongs to $\mathrm{CP}$ if one of the distances between the line segment $\mathrm{C}_{k-1} \mathrm{C}_{k}$ and each point (from $\mathbf{P}_{i}$ to $\mathbf{P}_{j}$ ) is greater than the precision value $\varepsilon$. This gives many critical points on curves and few critical points on straight lines. The compression rate is the ratio $k / j$.

- Frame template. The file describing the bottom of the frame slot proceeds from an automatic 2D digital frame tracer which is used to drive digital glasses edges. The model is illustrated in Figure 3. It has a total of 8 parameters. The model is overlaid on the representation of the image. For each variation of parameters, the correlation between the representation of the image and the points of the model is computed. The best position is reached when the correlation is maximal.

\section{Results}

Our algorithms qualification is focused on processing times and precise features extraction. 8 series of 10 images are made. For each series, the patient wears the same glasses frame. For each image, all parameters (eye centres and frame features) were determined with the help of a PC mouse and considered as references for the algorithms qualification. After few experimentation, the vectorization precision is set to $0.02 \mathrm{~mm}$. It is the best trade-off between results precision and processing times for our application. It allows to compress the frame pattern with a ratio equal to 12 . 


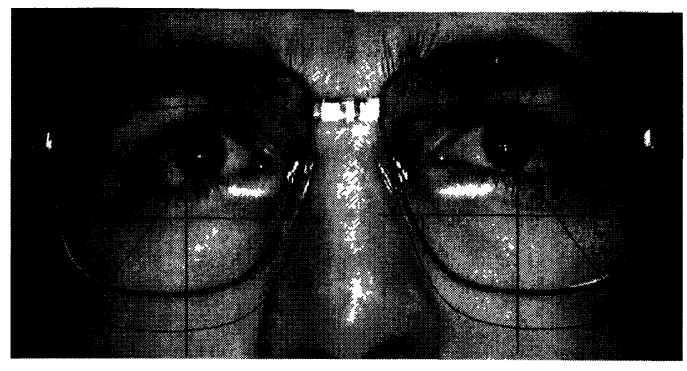

a)

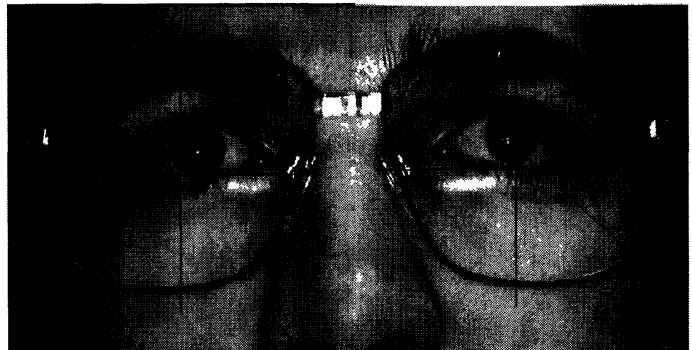

b)

Fig. 8. - a) Matching in progress. Reconstructed vectorized model during correlation. b) Model matched on frame image.

The matching algorithm required a lot of time to be fitted to our application. The algorithm optimization is based on the knowledge of the intervals of variation for the parameters. Test images were made to isolate each parameter and know their influence on matching operation. The matching by translation has 2 steps: a fast one $(6 \times 6$ matrix scan with a 5 pixels shift $)$ followed by a precise one $(6 \times 6$ matrix scan with a 1 pixel shift $)$. The last part of the processing is to adjust the frame model to the representation of the image by rotating $\left(\mathbf{R}_{x}, \mathbf{R}_{y \mathrm{r}}\right.$ for right side and $\mathbf{R}_{y 1}$ for left side). Our automatic extraction method reaches a success rate of $82 \%$ with a 2-pixels precision (see Figs. 8a, 8b). The success rate for the frame feature extraction is $92.5 \%$ for a difference of 3 pixels or less between the detected parameter and the original frame feature, which corresponds to the tolerance required for the measure.

\section{Conclusion}

The design of a full embedded system for parameters extraction, related to an optician dedicated application, was presented, and precise results obtained from optical point of view. The automatic frame extraction process appears to be the most accurate when based on a simplified representation of the image and an optimized frame model to be matched on the image. By reducing the variation for the parameters concerned by the matching process, we obtain a 92.5 percentage of success rate in this case.

\section{Acknowledgements}

The authors cordially thank Mr A.Chansavoir, former R \& D Manager of Instruments Dpt, for his helpful contribution to the co-operation between Essilor International Company and "École Supérieure de Chimie Physique Électronique" (CPE-Lyon). The authors wish also to acknowledge useful participation of Mrs H. Sottocasa.

\section{References}

[1] Bouyanzer H., Automatic features extraction on color images, application to parameters measurement, Thèse de doctorat de l'Université de Rouen (1992).

[2] Rosenfeld A. and Kak A.C., Digital picture processing, Vol.2 (Academic Press, New-York, 1992) 2nd edition. 
[3] Canny J., A computational approach to edge detection, IEEE Trans. Patt. Analysis Machine Intelligence 8 (1986) 679-698.

[4] Deriche R., Fast algorithms for low level vision, IEEE Trans. Patt. Analysis Machine Intelligence 12 (1987) 167-187.

[5] Sahoo P.K., Soltani S., Wong A.K. and Chen YC, A survey of thresholding techniques, Comput. Vision, Graph. Image Proc. 41 (1988) 233-260.

[6] Hong Park R. and Young Choi W., A new interpretation of the compass gradient edge operators, Comput. Vision, Graph. Image Proc. 47 (1989) 259-265.

[7] Pratt W.K., Digital image processing (Wiley Interscience, 1991) 2nd edition, pp. 449-489.

[8] Giraud N., Grisel R. and Ducarouge C., Tangents extraction method based on mathematical morphology operators, application to optical metrology, Proceedings of the 5th ICSPAT, Dallas (1994) pp. 11631167.

[9] Serra J., Image Analysis and Mathematical Morphology (Academic Press, London, 1982).

[10] Burr D.J., Elastic matching of lines drawing, IEEE Trans. Patt. Analysis Machine Intelligence 3 (1981) 708-713.

[11] Kass M., Witkin A. and Terzoupoulos D., Snakes: Active contour model, Proceedings of the 1st International Conference on Computer Vision (London, 1987).

[12] Yuille A., Hallinan P. and Cohen D., Feature extraction from faces using deformable templates, Int. J. Comput. Vision 8 (1992) 99-111.

[13] Horaud R. and Monga O., Vision par ordinateur - outils fondamentaux (Hermès, Paris, 1993) pp. 93102. 